\title{
Gain Enhancement of Monopole Antenna using AMC Surface
}

\author{
Faiza Mouhouche, Arab Azrar, Mokrane Dehmas, Kahina Djafer \\ University M'Hamed Bougara of Boumerdes, Institute of Electrical and Electronic Engineering, Signals and Systems \\ Laboratory, Department of Electronics, 35000 Boumerdes, Algeria. \\ corresponding author, E-mail: mouhouche35 @gmail.com
}

\begin{abstract}
A CPW rectangular-ring antenna over an Artificial Magnetic Conductor (AMC) is presented in this work. The AMC is a designed as a dual-band structure having an array of unit cells and operates at $2.45 \mathrm{GHz}$ and $5.20 \mathrm{GHz}$. A CPW antenna uses this dual-band AMC structures as a back-plane. Performance comparison is carried out with and without incorporation of AMC. The simulated and measured results show that the combination of the AMC reflector and the antenna provide directional properties at both frequency bands. It has been found that the antenna gain increases by about $5 \mathrm{~dB}$
\end{abstract}

\section{Introduction}

Microstrip antennas is an attractive solution in the design of modern wireless communication systems due to their many advantages solution to compact and ease-low-cost as light weight, low volume, low profile and planar configuration. However, Microstrip antennas suffer from a number of disadvantages as compared to conventional non-printed antennas. Some of their major drawbacks are the narrow bandwidth, low gain, and surface wave excitation that reduce radiation efficiency. To overcome the gain and efficiency a new design methodology is used which is an artificially created structures called metamaterial.

Metamaterial is an artificial resonant structure that is designed to obtain specific characteristics which are not naturally occurring in nature [1]. These unique characteristics of metamaterial have been used extensively in antennas and microwave applications in order to improve the desired performances [2]. The artificial magnetic conductor (AMC) is a type of metamaterial which introduces an in-phase reflection within the band gap of a desired frequency. AMC surface, also called the meta-surface, high-impedance surface (HIS), or reactive impedance surface, has been widely used as an artificial ground plane or reflector to enhance performance of many different antenna types while achieving profile miniaturization [3].

An artificial magnetic conductor (AMC) surface was proposed by Sievenpiper [4] and has widely investigated [5, 6]. An AMC works as a perfect magnetic conductor (PMC) at resonant frequencies with the $0^{\circ}$ phase of the reflected wave when illuminated by a normally incident plane wave. Its bandwidth is defined as the frequency range over which the phase of the reflection coefficient is between $\pm 90^{\circ}$ [7]. In contrast, a perfect electric conductor (PEC) is typically used as a reflectors to enable antenna radiation to focus in one direction. However, the use of PEC produces an image current that flows in the opposite direction relative to the original current. The image current will interfere with the original current, thereby attenuating or even cancelling the latter and consequently degrading the radiation efficiency. The attenuation can be reduced by separating the PEC surface from the antenna with a distance of $\lambda / 4$. However, the penalty for reducing the attenuation is the increase in the overall antenna dimension; hence, the design will not be low-profile, which is a highly desirable characteristic for communication devices.

Interestingly, the image current problem can be solved by utilizing a PMC, which produces an image current in the same direction as that of the original current. This implies that the reflection phase is $0^{\circ}$ and the magnitude of reflection coeffcient equals +1 . However, a PMC does not exist in nature, so an AMC can be designed only within a limited frequency band. AMC behaves like a PMC in the designed band, while it exhibits PEC characteristics in other bands. Many works of dual-band AMC structures have been proposed in literature, square with circular ring as slot, which is the classical structure $[8,9,10]$.

In this paper, we present a novel dual-band AMC structure that allows easy control of the first and second resonance frequency ratio, this achieved by inserting triangular slot into square metallic patch on grounded substrate. The proposed AMC structure is used as a reflector with CPW rectangular ring antenna. We verified the dual-band characteristics and enhancement of the radiation characteristics by fabricating and analyzing a CPW rectangular ring antenna over the AMC surface.

\section{Design and configuration}

For the dual-band 2.4/5.2 GHz WLAN application, a rectangular ring antenna with coplanar feed line $(\mathrm{CPW})$ using the substrate FR-4 with relative dielectric permittivity of 4.3 , loss tangent of 0.0017 and thickness of $h=1.62 \mathrm{~mm}$ is designed as shown in Figure 1. The parameters of the antenna are shown in Tab. 1 so that the width of the feed line, the gap between the feed and rectangular ground have been selected so that the input impedance is $50 \Omega$. 

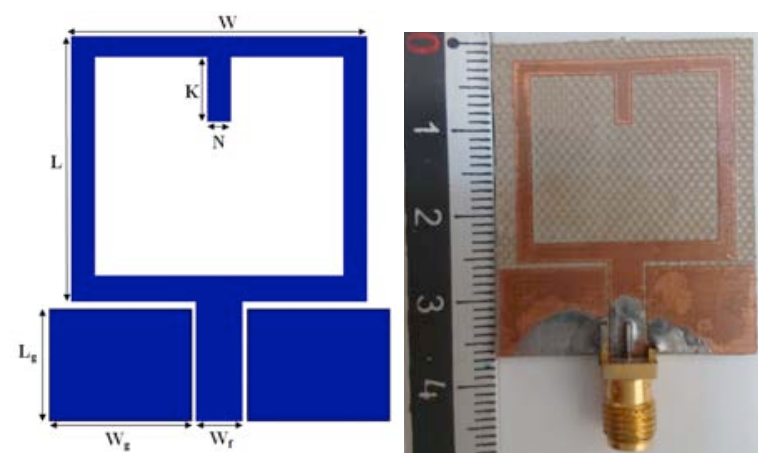

Fig .1. a) Geometry and dimensions of CPW-fed rectangular-ring antenna, b) Photograph of the fabricated antenna.

\section{Table. 1: AMC unit cell dimensions}

\begin{tabular}{|c|c|c|c|c|c|c|c|}
\hline Parameters & $\mathrm{L}$ & $\mathrm{W}$ & $\mathrm{K}$ & $\mathrm{N}$ & $\mathrm{Wf}$ & $\mathrm{Wg}$ & $\mathrm{Lg}$ \\
\hline $\begin{array}{c}\text { Values } \\
(\mathrm{mm})\end{array}$ & 23.5 & 27 & $\begin{array}{c}6 . \\
8\end{array}$ & 2 & 4.08 & $\begin{array}{c}12.5 \\
6\end{array}$ & 10 \\
\hline
\end{tabular}

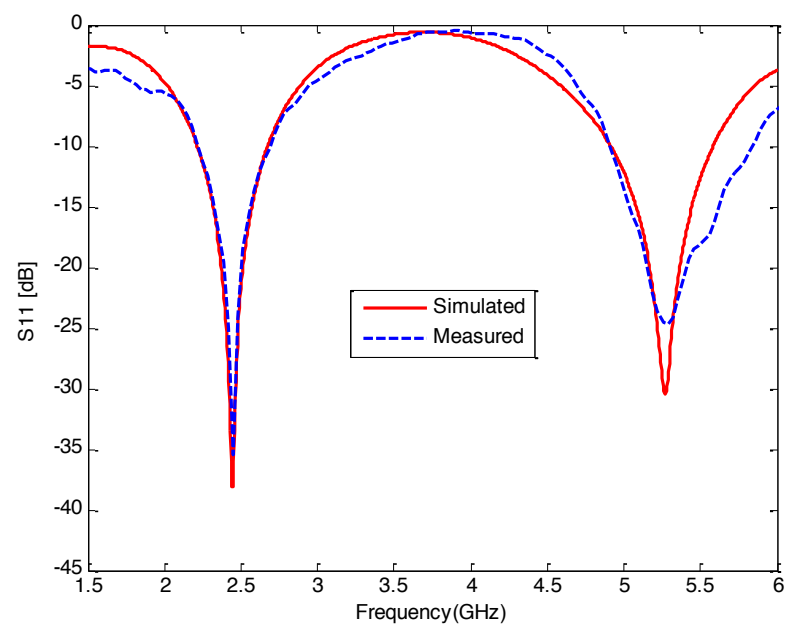

Fig .2. Simulated and measured return loss of CPW antenna.

The dual-band antenna has been constructed and experimentally studied. The simulated and measured return losses obtained by using software simulator and Roche \&Schwarz; Znb20: Vector Network Analyzer: $10 \mathrm{KHz}-20 \mathrm{G}$ $\mathrm{Hz}$ are presented respectively. Figure 2 shows the measurement and simulation frequency responses of the return loss for the CPW antenna. It is noted that the CPW antenna has dual-band characteristic. The simulation data shows that the low-band resonant frequency locates at about $2.44 \mathrm{GHz}$, with the $-10 \mathrm{~dB}$ impedance bandwidth from 2.21 to $2.67 \mathrm{GHz}$. The high-band resonant frequency locates at about $5.27 \mathrm{GHz}$, with the $-10 \mathrm{~dB}$ impedance bandwidth from 4.91 to $5.58 \mathrm{GHz}$. The antenna covers WLAN standard $(2.4 \mathrm{GHz}, 5.2 \mathrm{GHz})$.

The simulated antenna radiation patterns at 2.44 and 5.27 $\mathrm{GHz}$ are plotted in Figure 3. It is observed that the E-plane radiation pattern at $2.44 \mathrm{GHz}$ is bidirectional, while at 5.27
$\mathrm{GHz}$ the radiation patterns exhibit broadside radiation characteristics. In addition, antenna gains of about $2.72 \mathrm{dBi}$ at $2.44 \mathrm{GHz}$ and $3.45 \mathrm{dBi}$ at $5.27 \mathrm{GHz}$.

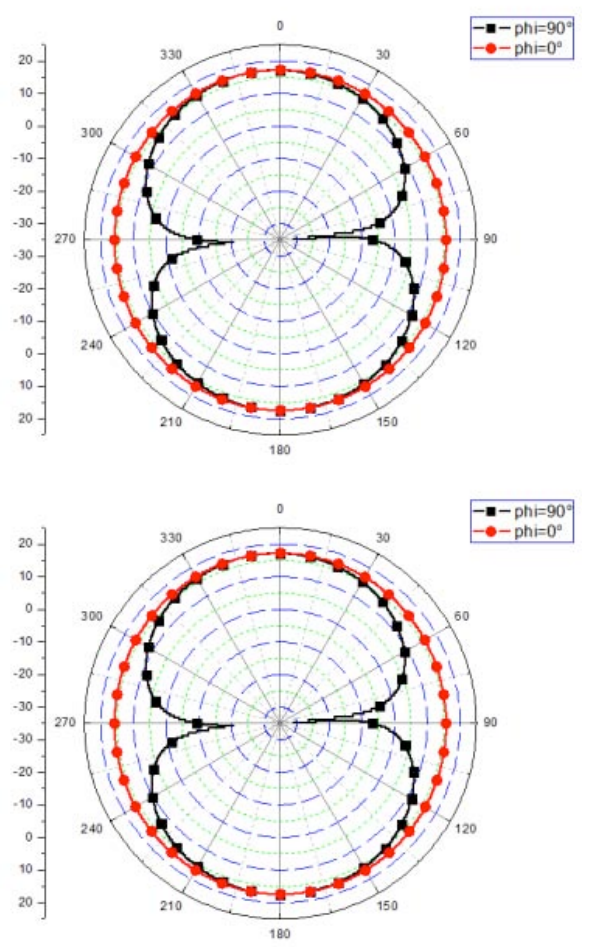

Fig.3. Simulated radiation pattern of E-plane of the proposed antenna at a) $2.44 \mathrm{GHz}$ b) $5.27 \mathrm{GHz}$.

\section{AMC design}

There are many candidate structures for the design of a dual in-phase reflection phase band AMC. In this paper, a novel structure of the AMC is proposed to operate at $2.45 \mathrm{GHz}$ and $5.2 \mathrm{GHz}$. The structure is printed on a FR-4 substrate with a thickness $1.62 \mathrm{~mm}$, relative permittivity of 4.3, and loss tangent of 0.0017 . The other side of the substrate is the metallic ground plane, as depicted in Figure 4.

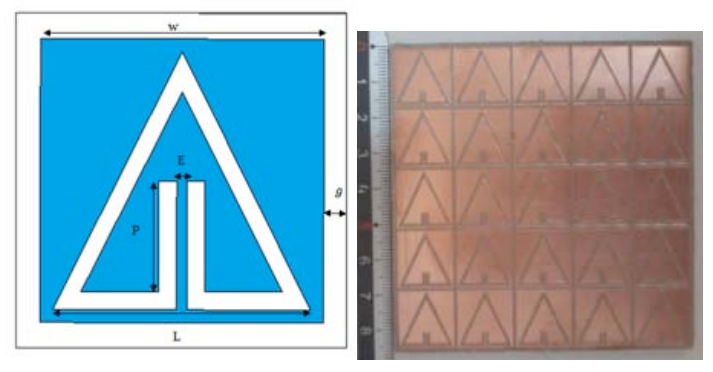

Fig .4. Configuration of the AMC structure under investigation, a) the $\mathrm{AMC}$ reflector b) unit cell

Table.2: AMC unit cell dimensions

\begin{tabular}{|l|l|l|l|l|l|}
\hline Parameters & L & W & E & P & g \\
\hline Values(mm & 15.25 & 16.25 & 0.4 & 4 & 0.5 \\
\hline
\end{tabular}


The simulation of the AMC structure is carried out using a waveguide setup to mimic the infinite array of AMC structure. The setup is designed in CST software using parallel E-plane and H-plane boundary condition and the system is excited using a waveguide port placed on the top of the waveguide and de-embedded to the surface of AMC structure, as shown in Figure 5.

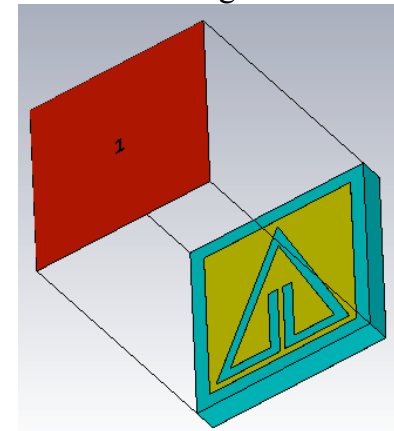

Fig .5. Waveguide simulation setup of proposed AMC unit cell.

\subsection{Parameter study}

For further study, the effect of square patch and parameters of the slot are discussed. Firstly, the effect of varying L from $14.25 \mathrm{~mm}$ to $16.25 \mathrm{~mm}$ with all other dimensions remaining the same as is shown in Figure 6 (a). The reflection phase curves change intensely with the length $\mathrm{L}$ in the band of $2.45 \mathrm{GHz}$ and $5.2 \mathrm{GHz}$. Both lower and higher resonant frequency points move toward the lower frequency band as the length $\mathrm{L}$ increases.

Secondly, the Figure 6(b) shows simulated reflection phase response curves for the proposed structure with different dimensions of P. It can be seen that at the reflection phase is unvaried in the band around the frequency of $2.45 \mathrm{GHz}$; while within the band around the frequency of $5.2 \mathrm{GHz}$ it exhibits a noteworthy difference. In this band, the resonance frequency increases with the increase of the parameter P. Figure 6(c) shows the reflection phase curves when the gap g width varies. It can be seen that the curves change significantly in the band around the frequency of $2.45 \mathrm{GHz}$ and slightly the band around the frequency of $5.20 \mathrm{GHz}$.

Based on the analysis of the above parametric study, the AMC reflector can be designed using the optimized values of the parameters listed in Table. 2. The final reflection phase versus frequency is depicted in figure 7 . As we can see in figure, the reflection phase frequency band of this AMC surface is very narrow about $80 \mathrm{MHz}$ in the frequency band centered at $2.45 \mathrm{GHz}$ and $244 \mathrm{MHz}$ in the frequency band centered at $5.20 \mathrm{GHz}$.

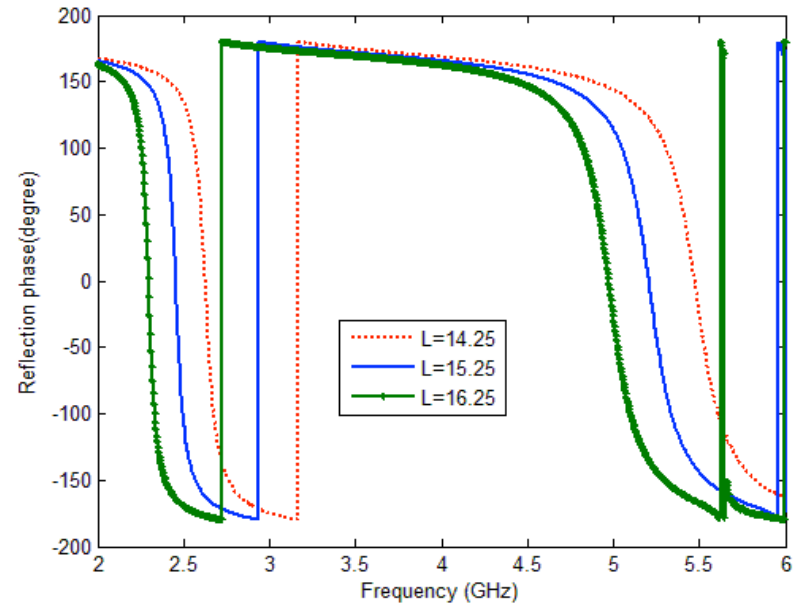

(a)

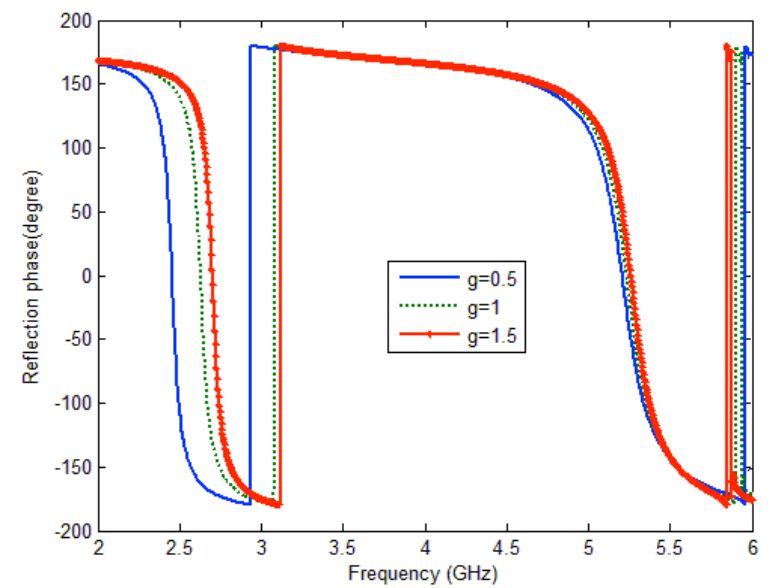

(b)

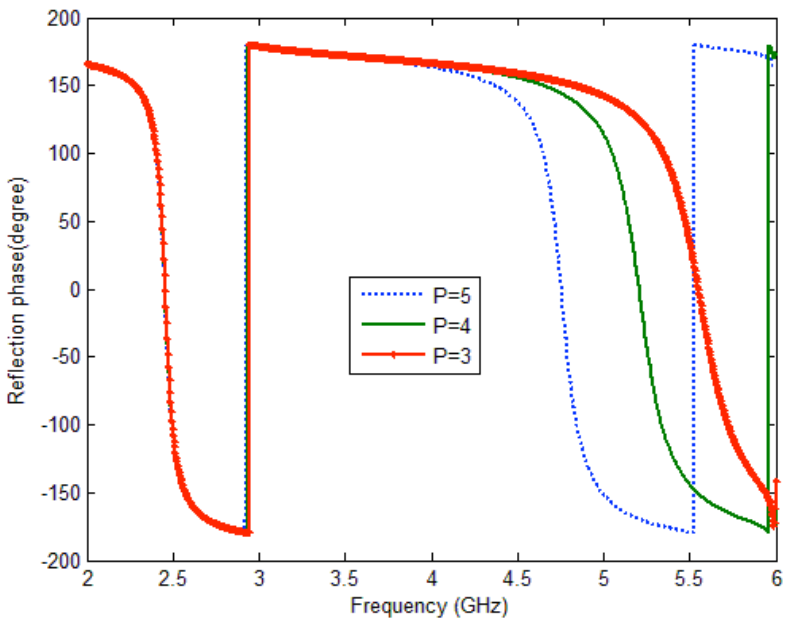

(c)

Fig .6. Simulation reflection phase a) varying the (c) length of slot $L, b$ ) varying $p, c)$ varying the gap 


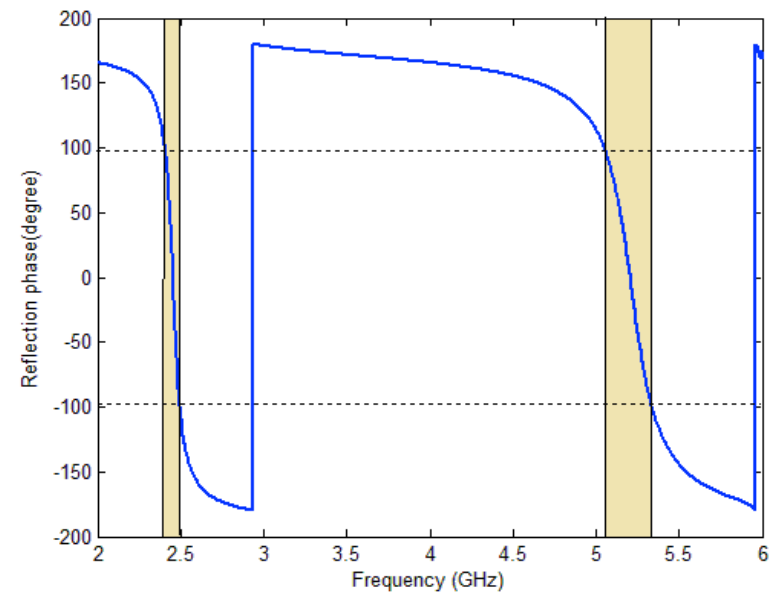

Fig. 7. Reflection phase response for proposed AMC structure.

Figure 8 shows the simulated current distribution on the metallic patch surface of the AMC structure at $2.45 \mathrm{GHz}$ and $5.20 \mathrm{GHz}$ frequencies taken from the first and second reflection phase bands, respectively. From the figure, it is obvious that there is more current on the square patch at $2.45 \mathrm{GHz}$.Similarly, there is a dense of current in the inner triangular metallic region at $5.20 \mathrm{GHz}$. This indicates that the first frequency is generated by the entire square patch , whereas the second frequency by the center triangular metallic region.

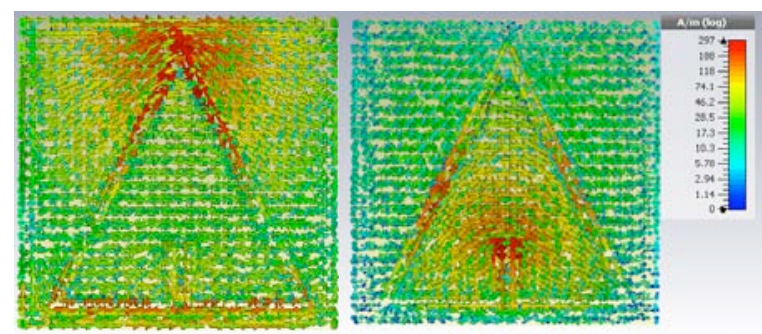

Fig .8. Surface current distribution of the dual band AMC structure at (a) $2.45 \mathrm{GHz}$, (b) $5.20 \mathrm{GHz}$.

\section{Antenna with AMC structure}

In the final structure, a CPW antenna is incorporated with an array of metal triangular patch cells. The overall structure size is $86.25 \times 86.25 \mathrm{~mm}^{2}$. The antenna is put in the middle of the reflector and height of $\mathrm{d} \mathrm{mm}$ above the reflector. A thin foam layer with thickness $d=12 \mathrm{~mm}$, relative permittivity of 1.01 , and loss tangent of $1.1 \times 10^{-4}$ is placed between the AMC reflector and the CPW antenna for measurement as illustrated in Figure 9. This configuration can significantly reduce the spacing between the antenna and the reflector, and provide directional function for both frequency bands.

Figure 10 illustrates the simulated and measured return losses of CPW antenna with AMC reflector. Here the antenna is situated at $\mathrm{d}=12 \mathrm{~mm}$ (about 0.07 wavelength) above AMC reflector. We can notice that the simulated results agree with measured results. The little difference in bandwidth between simulated and measured may be caused by the fabrication tolerance or the external effects during the measurements.
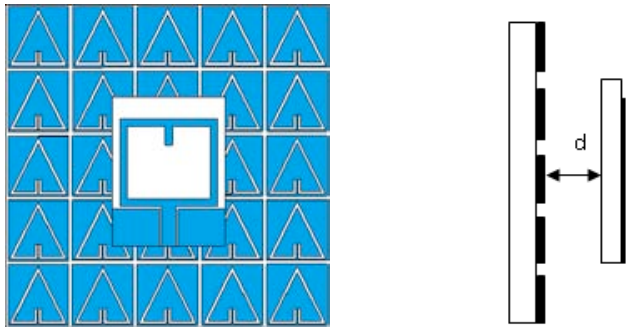

(a)
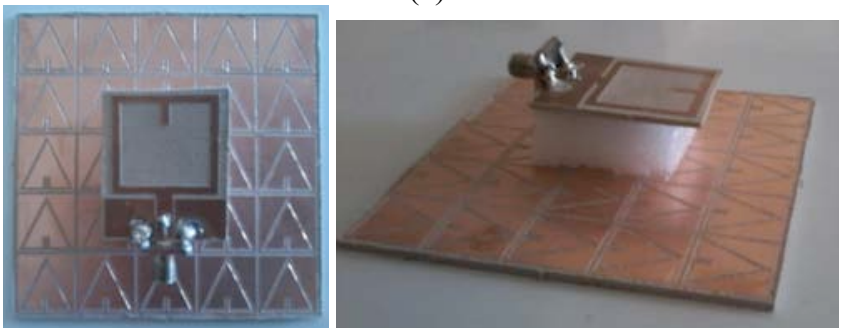

(b)

Fig .9. a) Configuration of cpw antenna with an AMC reflector, b) Photograph of the fabricated AMC with antenna on top: left (top view) and right (side view).

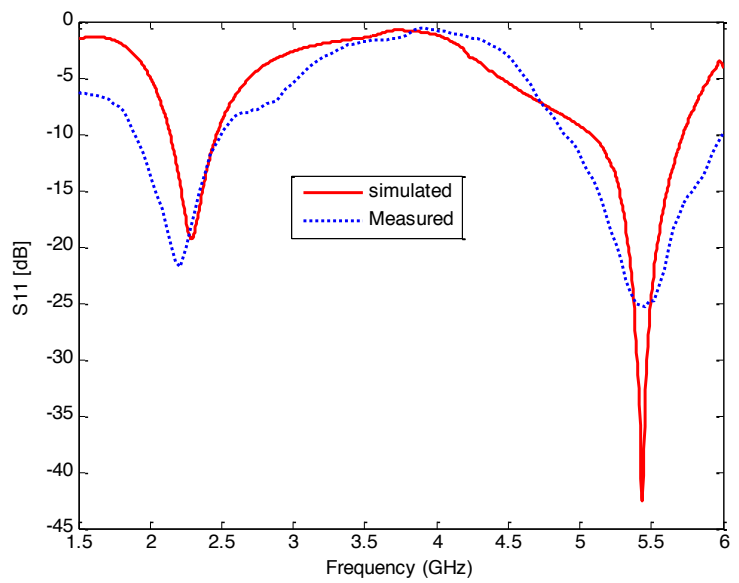

Fig .10. Measured return loss of the antenna with AMC in comparison to the antenna in free space.

Figure 11 depicts the measured cross and co-polar radiation patterns for the CPW antenna with AMC design at $2.44 \mathrm{GHz}$ and $5.27 \mathrm{GHz}$. It is observed that the CPW antenna alone can radiate bidirectional. While the antenna with AMC reflector shows unidirectional radiation pattern with small back lobes.

For comparison, a similar model that the cpw antenna integrated with a metal reflector (antenna over PEC) and antenna with AMC over a large metal as depicted in Figure 12 .It can be seen that it is hard for the antenna over PEC to work at the two frequency bands properly, whereas for AMC antenna over large metal $(300 \mathrm{~mm} \times 300 \mathrm{~mm})$ as depicted in 
Figure 13, the results is the same as the antenna over AMC in free space in both resonance frequencies.

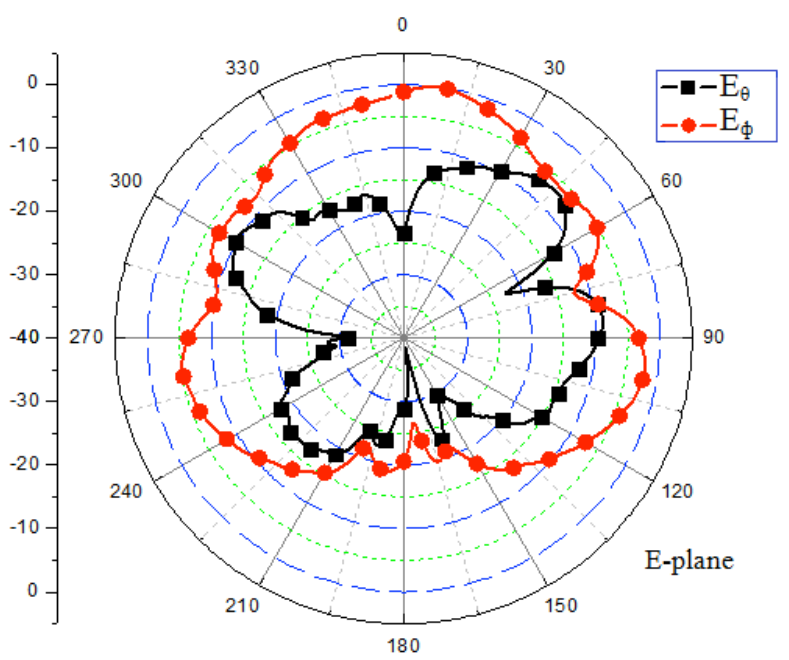

(a) at $2.44 \mathrm{GH}$

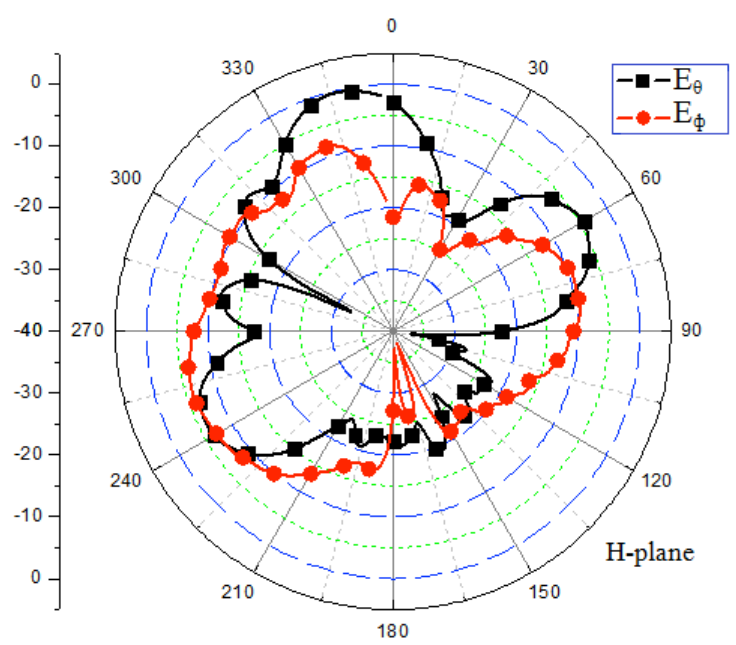

(b) at $2.44 \mathrm{GHz}$

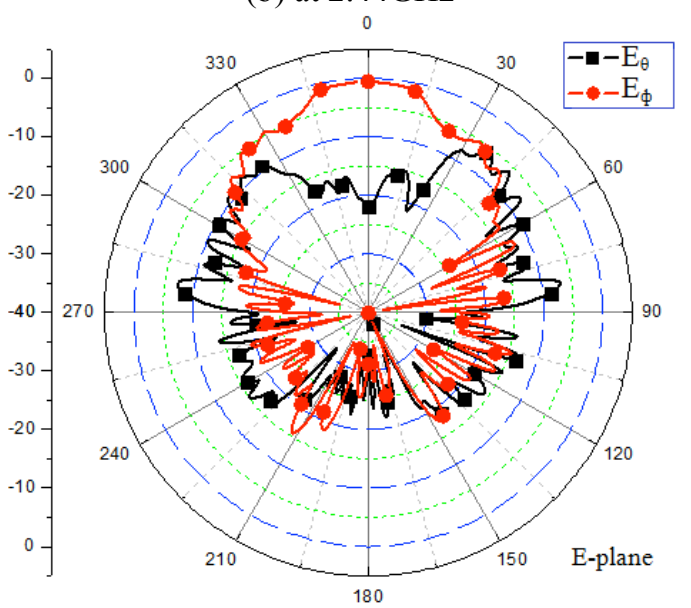

(c ) at $5.27 \mathrm{GHz}$

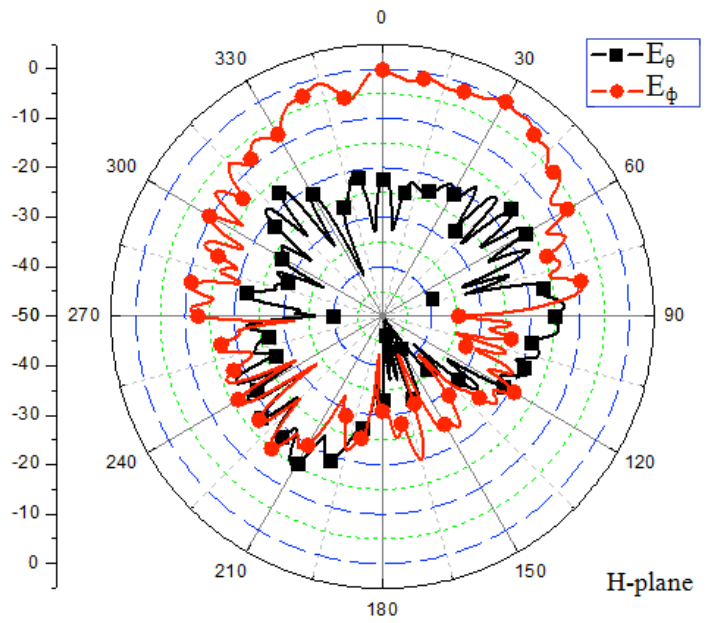

(d) at $5.27 \mathrm{GHz}$

Fig. 11. Measured radiation patterns of antenna integrated with $\mathrm{AMC}$ at a) $2.44 \mathrm{GHz}$, b) $5.27 \mathrm{GHz}$.

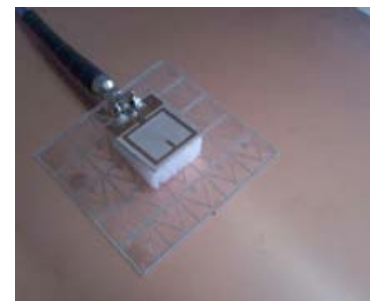

Fig.12. Measurement setup for the proposed antenna over large metallic surface

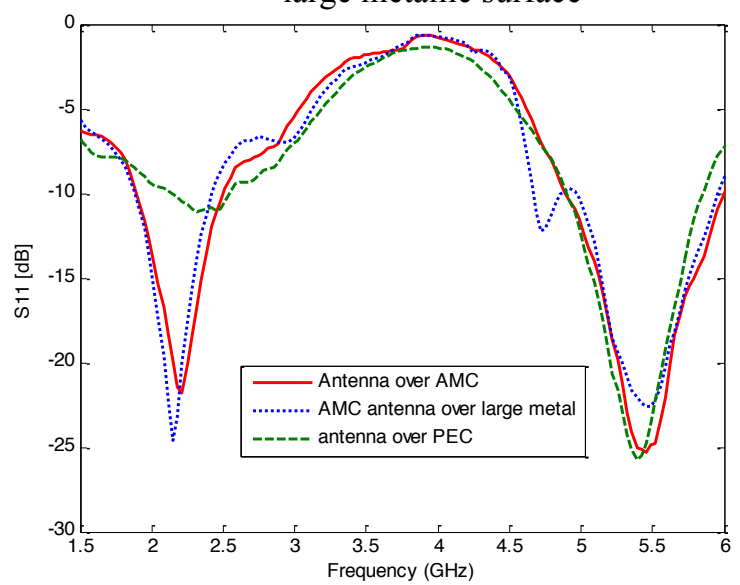

Fig .13. Measured return loss for various cases

The simulated and measured gain performances of antenna alone and antenna with AMC for two operating bandwidth are illustrated Table. 3.The cpw antenna gain is about 2.72 and $3.45 \mathrm{~dB}$ for $2.44 \mathrm{GHz}$ and $5.27 \mathrm{GHz}$.With $\mathrm{AMC}$ reflector, the measured antenna gain is improved by factor of 5.17 and $5.75 \mathrm{~dB}$ for lower and higher operating frequencies . It can be noticed that the gain and directivity increase by using AMC reflector . 
Table. 3: Simulated antenna performance

\begin{tabular}{|l|l|l|l|l|}
\hline \multirow{2}{*}{ Antenna } & \multicolumn{2}{|l|}{$\mathrm{f}=2.44 \mathrm{GHz}$} & \multicolumn{2}{l|}{$=5.27 \mathrm{GHz}$} \\
\cline { 2 - 5 } & $\begin{array}{l}\text { Gain } \\
(\mathrm{dB})\end{array}$ & $\begin{array}{l}\text { Directivity } \\
(\mathrm{dB})\end{array}$ & $\begin{array}{l}\text { Gain } \\
(\mathrm{dB})\end{array}$ & $\begin{array}{l}\text { Directivity } \\
(\mathrm{dB})\end{array}$ \\
\hline $\begin{array}{l}\text { Simulated } \\
\text { CPW-ant }\end{array}$ & 2.72 & 2.85 & 3.45 & 4 \\
\hline $\begin{array}{l}\text { Measured } \\
\text { CPW-ant } \\
\text { with AMC }\end{array}$ & 7.89 & 8.18 & 9.20 & 9.72 \\
\hline
\end{tabular}

\section{Conclusion}

The dual-band AMC structure as a reflector for the CPW rectangular ring antenna is investigated. The radiation performances, including antenna gain, directivity and radiation pattern are significantly improved as a contrast to the reference coplanar antenna. The gain enhancement can be up to more than $5 \mathrm{~dB}$. The investigations show that when antenna with AMC reflector is placed over large metallic plate, the performances of the antenna are not changed as compared to the isolated antenna alone.

\section{References}

[1] A. Kumar, and D. Kumar V., " High-performance Metamaterial Patch Antenna", Microwave and Optical Technology Letters, vol. 55 February( 2013), 409-413.

[2] P. Prakash, M. P. Abegaonkar, A. Basu, and S. K. Koul, "Gain enhancement of a CPW-fed monopole antenna using polarization-insensitive AMC structure," IEEE Antennas and Wireless Propagation Letters, vol. 12, (2013). 1315-1318

[3] R. C. Hadarig, de Cos, M.E., and Las-Heras, F, "Novel Miniaturized Artificial Magnetic Conductor," Antennas and Wireless Propagation Letters, IEEE, vol. 12, January (2013), 174-177.

[4] L.Z.D.Sievenpiper,R.F.J.Broas,N.G.Alexopolous,

E.Yablonovitch, " High-impedance electromagnetic surfaces with a forbidden frequency band", IEEE Trans. Microw. Theory Techn., vol. 47, November (1999),2059-2074.

[5] H. T. Qun Luo, Zhitong Huang, Xudong Wang, Zheng Guo, and Yuefeng Ji, "Unidirectional DualBand CPW-Fed Antenna Loaded with an AMC Reflector", International Journal of Antennas and Propagation, (2013).

[6] H. S. S. a. M. K. M. Gaurav Kumar Pandey, "Platform tolerant UWB antenna over multi-band AMC structure," Microwave and Optical Technology Letters, vol. 58, March (2016), 1052-1059.

[7] K. S. H. Mosallaei, "Antenna miniaturization and bandwidth enhancement using a reactive impedance substrate " IEEE Transactions on Antennas and Propagation, vol. 52, September (2004), 2403 - 2414.

[8] N.A.Abbasi R.J. Langley, "Multiband-integrated antenna / artificial magnetic conductor" IET Microwaves, Antennas \& Propagation, vol. 5, (2011), 711-717.

[9] X. Mu, W. Jiang, S.-X. Gong, and F.-W, " DualBand Low Profile Directional Antenna with High Impedance Surface Reflector", WangProgress In Electromagnetics Research Letters, Vol. 25, (2011), 67-75.

[10] Mohammed Amin Meriche, Hussein Attia , Abderraouf Messai and Tayeb. A. Denidni."Gain Improvement of a Wideband Monopole Antenna with Novel Artificial Magnetic Conductor"IEEE conference paper, July 2016 